\title{
Imaging in carcinoma of ovary
}

\author{
SANJAY THULKAR
}

Role of imaging in ovarian cancer is to detect and characterize adnexal masses, recognize unusual findings that may suggest atypical or alternative diagnosis, demonstrate metastases in order to prevent surgical understaging and detect specific sites of the disease that may be unresectable ${ }^{1}$.

\section{ULTRASONOGRAPHY}

Ultrasonography is the first imaging modality to diagnose or confirm the clinically suspected adnexal mass. It is also used to characterize the adnexal masses based on their morphological features. Transvaginal sonography is better than trans abdominal approach for characterization of adnexal masses. Large, bilateral, complex solidcystic masses are usually malignant. Cysts with thick walls, irregular thick septation (more than $3 \mathrm{~mm}$ ), papillary projections or mural nodules favor malignancy. Ovaries enlarged for the age are also suspicious for malignancy. Presence of ascites, peritoneal deposits and lymph node enlargement are other supportive evidences. Physiological hemorrhagic cysts may sometimes have suspicious appearances on ultrasound and hence it is important to follow these with ultrasound and only persistent lesions should be considered abnormal ${ }^{2}$.

Very high accuracy of Doppler studies was reported earlier for the diagnosis of malignant ovarian masses ${ }^{3}$, however, subsequent studies found that similar Doppler findings may also be seen in inflammatory diseases and physiological cysts and hence, characterization of the adnexal masses based on Doppler studies only is unreliable. Combined morphological and Doppler sonographic evaluation provides the best results in detection of ovarian cancer ${ }^{4}$.

\footnotetext{
Department of Radioagnosis

Institute Rotary Hospital Centre, All India Institute of Medical Sciences, New Delhi-29

E-mail: thulkar@hotmail.com
}

\section{CT Scan}

CT is the main stay of preoperative evaluation of ovarian cancer. The CT features of ovarian cancer show varied morphological patterns: a multi locular cyst, thick wall or internal septations, solid mural nodules, complex cysticsolid mass or lobulated papillary mass.

Ancillary findings of malignancy such as ascites, peritoneal deposits, lymphadenopathy and pleural effusion may also be present. Peritoneal deposits are seen as soft tissue nodules or plaque like thickening and enhancement of the peritoneum. The sensitivity of CT in detection of peritoneal deposits is moderate; conventional CT scanners detected only up to $50 \%$ of peritoneal deposits that are $5 \mathrm{~mm}$ or less in size. The multi detector CT scanners have improved sensitivity in detection of small peritoneal deposits, especially in upper abdomen ${ }^{5}$.

Omental deposits are initially seen as multiple nodular lesions in omental fat or frank 'omental cake', which is usually seen anterior to transverse colon or small bowel. Involvement of uterus, rectum, colon and small bowel by the tumour is well demonstrated on CT. CT accurately detects para aortic and pelvic lymph node enlargement. Intra hepatic or splenic metastases are rare and occur late in the course of the disease.

Preoperative CT can also accurately predict the surgical outcome and hence has important role in deciding the management of ovarian cancer. Findings that suggest unresectable disease include greater than 1-2 cm deposits at porta hepatis, fissure for ligamnetum teres, GB fossa, diaphragm, gastrohepatic ligament, lesser sac and root of the mesentery. Invasion of pelvic sidewall and ureters as well as retroperitoneum lymphadenopathy encasing aorta or located above the renal hila also suggest unresectable disease $^{6,7}$. Detection of distant metastases on CT 
help to prevent surgical under staging, although it does not necessarily makes the patient inoperable. In few other patients CT may identify different primary site with metastases to ovary and hence change the management. Metastatic ovarian carcinoma is often indistinguishable from primary ovarian carcinoma; although multilocularity on ultrasound or MRI is uncommon with metastases.

\section{MRI}

The morphological features of ovarian cancer on MRI are similar to those seen on sonography or $\mathrm{CT}$, but because of excellent soft tissue contrast, the details are better demonstrated. Most tumours are low or intermediate in signal intensity on $\mathrm{T} 1 \mathrm{~W}$ and high in signal intensity on $\mathrm{T} 2 \mathrm{~W}$ images. Contrast enhancement helps to differentiate solid component of the tumour from blood clot or debris which do not enhance. MRI is better than other modalities in determining the origin of a pelvic mass as well as its characterization into benign and malignant $t^{8}$. Hence, it is used as a problem solving tool in the settings of indeterminate mass on sonography ${ }^{2}$. MR is also accurate in demonstration of direct involvement of other pelvic structures by the ovarian tumour. Although CT is the primary imaging modality for preoperative staging, MRI may be equal to $\mathrm{CT}^{9}$. At present, use of MRI in ovarian cancer limited primarily because of long imaging time required for MRI evaluation of entire abdomen and pelvis, high cost and limited availability ${ }^{2}$.

\section{FOLLOW UP IMAGING}

Both CT and MRI are useful in detection of macroscopic recurrences. On CT and MRI, recurrences can be seen in various forms; pelvic or vault masses, peritoneal deposits, ascites, lymphadenopathy or rarely, hematogenous metastases to various distant organs. The recur- rences are also common at the site of previous lesions hence; review of preoperative CT scans is desirable while evaluating the follow-up CT scans. Careful search for the recurrences should be made at these sites. The CT criteria suggesting unresectability of recurrent disease are same as those for primary disease. In addition, presence of pelvic sidewall invasion, hydronephrosis and bowel obstruction are important findings which may preclude the pelvic exenteration $^{10}$.

\section{REFERENCES:}

1. Cookley FV. Staging ovarian cancer: Role of imaging. Radiol Cin N Am 2002; 40: 609-36.

2. Mironov S, Akin O, Taskar NP, et al. Ovarian cancer. Radiol Clin N Am 2007;45:149-66.

3. Kurjak A, Zalud I, Alfirec Z. Evaluation of adnexal masses with transvaginal color Doppler ultrasound. $J$ Ultrasound Med 1991;10:295-97.

4. Kinkel K, Hricak $H$, Ying L, et al. US characterization of ovarian masses: a meta analysis. Radiology 2000;217:803-11.

5. Pannu HK, Horton KM, Fishman EK, et al. Thin section dual phase multidetetor row computed tomography detection of peritoneal metastases in gynecologic cancers. J Comput Assist Tomogr 2003;27:333-40.

6. Nelson BE, Rosenfield AT, Schwartz PE. Preoperative abdominopelvic computed tomography prediction of optimal cytoreduction in epithelial ovarian carcinoma. J Clin Oncol 1993; 11: 166-72.

7. Qayyum A, Coakley FV, Westphalen AC, et al. Role of $C T$ and $M R$ imaging in predicting optimal cytoreduction of newly diagnosed primary epithelial ovarian cancer. Gynecol Oncol 2005;96:301-06.

8. Kurtz AB, Tsimikas JV, Tempany CM, et al. Diagnosis and staging of ovarian cancer: comparative values of Doppler and conventional US, CT and MR imaging correlated with surgery and histopathologic analysis -report of the Radiology Diagnostic Oncology group. Radiology 1999;212:19-27.

9. Tempany CM, Zou KH, Silverman SG, et al. Staging of advanced ovarian cancer: comparison of imaging modalities-report from Radiological Diagnostic Oncology Group. Radiology 2000;215:761-67.

10. Funt FA, Hricak $H$, Abu Rustum $N$, et al. Role of CT in the management of recurrent ovarian cancer. Am J Roentgenol 2004;182:393-98. 